NORTH

AMERICAN

PRIMARY CARE From the North American

RESEARCH $\quad$ Primary Care Research Group

Ann Fam Med 2006;4:566-567. DOI: 10.1370/afm.660.

\section{CLINICAL AND TRANSLATIONAL SCIENCE AWARDS: A POTENTIAL SOURCE OF SUPPORT FOR PRIMARY CARE RESEARCH}

Primary care researchers have long recognized the tremendous difficulties in translating research findings into practice. The huge gaps between what is theoretically possible and what is actually delivered in our health care system have been highlighted in multiple forums, including several reports from the Institute of Medicine. However, translational research faces many complex challenges, including increasing regulatory headaches, few educational programs to prepare researchers for this type of work, growing clinical service demands, and reduced financial margins that have cut protected research time for many clinical and translational researchers. In response to these and other related issues, the NIH developed the NIH Roadmap for Medical Research. One of the themes of the Roadmap Initiative has given rise to the new Clinical and Translational Science Awards, or CTSAs, aimed at reengineering the clinical research infrastructure at academic health centers to catalyze the development of and provide a definable academic home for "a new discipline of clinical and translational science." The NIH announcement indicates that this is being looked at as a "new program designed to transform clinical and translational research, so that new treatments can be developed more efficiently and delivered more quickly to patients."

Two Requests for Applications were released by $\mathrm{NIH}$ in October 2005, one funding 4 to 7 CTSAs starting in 2006 for a total of $\$ 30$ million and another with $\$ 11.5$ million in funding for up to 50 planning grants for academic centers not yet ready to put a full program together. There will not be any further planning grant cycles, but it is anticipated that the number of full CTSA awards will increase each year so that by 2012, 60 CTSAs will receive approximately $\$ 500$ million per year. These awards take the place of the General Clinical Research Center program, with a gradual phasing out of the GCRCs over the next few years. Clinical research is defined by the CTSA program as including studies that involve human subjects. Translational research is defined as having 2 components: (1) the process of applying discoveries made in labs, testing them in animals, and developing studies in humans, and (2) research aimed at enhancing the adoption of best treatment practices into the medical community. CTSAs have to involve an educational component, training new clinical and translational researchers. Linkages to the community and to health care providers are also stressed as a part of this new initiative. Further information on CTSAs can be found at http:// www.ncr.nih.gov/clinicaldiscipline.asp.

What does this have to do with NAPCRG and those of us in primary care research? It is hard to tell how this initiative will pan out over time, and many of the CTSA applications will likely have little or no input or involvement from primary care researchers. However, the CTSAs could be a tremendous opportunity for those of us in academic medical centers to plug into the research enterprise in ways that we have not dreamed of previously. Our researchers, often more than anyone else, have forged connections with the community and with networks of practices and clinicians. In our community participatory research and our practice based research networks, we have established the bidirectional communications between researchers and the community highlighted in the background materials for the CTSA. We have focused a lot of attention on research aimed at enhancing the adoption of best practices in the community and have a great deal of expertise to share. Our fellowships and other research training programs are already geared toward preparing young researchers for careers in this area and could provide valuable experience and infrastructure for the necessary educational component of the CTSA. Our academic medical centers need us to truly accomplish the goals set out in the CTSA RFA and the accompanying materials - whether they know it or not. It is our job to educate both our academic centers and NIH. I strongly encourage our primary care researchers and other leaders in academic medical centers to plunge into the research politics of your home institution and start the educational process, if you haven't already done so. There may be a steep learning curve for everyone, but the potential benefits at the end of the process - with much needed infrastructure funding and stronger links to the rest of the research enterprise-are worth it.

NAPCRG will be paying a good bit of attention to our members' experiences with CTSAs over the next few years, as this is potentially an important development. NAPCRG partnered with the Association of Departments of Family Medicine on a survey of researchers and chairs who submitted proposals in the first round of applications. NAPCRG also offered a special session at the 2006 Annual Meeting designed to share information and the experiences of the applicants. I hope to see some stimulating discussions of 
this topic at our meetings, as NAPCRG can serve as a source of information and support for our members as they try to become a part of this effort to transform a portion of our research enterprise.

Perry Dickinson, MD, NAPCRG President

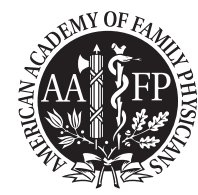

From the American Academy of Family Physicians

Ann Fam Med 2006;4:567-568. DOI: 10.1370/afm.657

\section{COLLABORATE, DON'T COMPETE, SAY FPS, PEDIATRICIANS}

A new article in Pediatrics written by staff members in the AAFP's Robert Graham Center in Washington notes that family physicians provide a medical home to about one third of the country's children "but face shrinking panels of children." Authors Robert Phillips, MD, MSPH, director of the Graham Center; other Graham Center staff members; and pediatrician Scott Shipman, MD, MPH, assistant professor at the Center for the Evaluative Clinical Sciences at Dartmouth Medical School, Hanover, NH, suggest that now is the time for collaboration (not competition) between family medicine and pediatrics.

Family physicians and pediatricians share common ground as they tackle issues such as children's unmet needs; health in the context of families and communities; and "millennial morbidities" such as violence, obesity, family distress and poverty says the abstract for "Family Physicians in the Child Health Care Workforce: Opportunities for Collaboration in Improving the Health of Children" in the September issue of Pediatrics.

Although children's visits to family physicians decreased by nearly 25\% from 1992 to 2002, "family medicine's role in children's health care is more stable in rural communities, for adolescents and for underserved populations," say the authors. "The growth of the pediatric workforce has largely occurred in areas of affluence and in urban or suburban areas. ... Rural and other underserved populations depend more on FPs."

Both pediatrics and family medicine "play an important role" in safety-net programs such as community health centers, "and health centers probably represent one of the best models of clinical cooperation between pediatricians and FPs in caring for communities," the authors say. "Health centers could be a laboratory for collaborative education."

In addition to pursuing advocacy efforts on behalf of children, the 2 specialties share a commitment to the concept of "medical home," which was formulated by the American Academy of Pediatrics (AAP) and was incorporated in the 2004 Future of Family Medicine report. Marking another common interest, the AAP

Task Force on the Family in 2003 concluded that children's outcomes were strongly influenced by how well their families functioned and that pediatricians could do much to help nurture and support families.

After reviewing commonalities among the 2 specialties, as well as threats to children's health, the authors say family medicine's options include

- relinquish clinical care of children to pediatricians and focus on working with internists to meet the increasing demands of aging adults;

- relinquish most clinical care of children and focus on preparing some FPs to care for children in rural and underserved areas;

- compete head-to-head with pediatricians, nursepractitioners (NPs) and physician assistants (PAs) for a shrinking child health care market; or

- seriously engage pediatricians, NPs and PAs in building "new models of training and practice that benefit from all sets of skill and compassion to provide better care in a family- and community-focused environment. This collaboration could involve joint or combined training and aggressive joint advocacy for improved services, both clinical and in the community."

The authors bid for the last option. "Stimulated by a shrinking market for providing care to individual children, FPs and pediatricians have an opportunity to join efforts in meeting the needs of children, for many of whom their most pressing morbidity risks are framed in the context of their families and communities," the authors say.

At least one pediatrician welcomes the authors' challenge. Richard Pan, MD, MPH, assistant professor of pediatrics and medicine at the University of California, Davis, in Sacramento wrote the commentary "A Jacobian Future: Can Everyone Have a Medical Home?" in the same issue of Pediatrics. An extract is available online without a subscription at: http://pediatrics.aappublications.org/cgi/content/extract/118/3/1254.

Pan notes the traditional "uneasy relationship" between FPs and pediatricians as competitors and partners in the care of children and adolescents. Also, he notes, "Pediatrics has recognized the importance of caring for families to improve child health, potentially leading to a collision course with family medicine."

"Phillips et al now bring a challenge to the pediatric community regarding the future of health care for children and adolescents," says Pan. He defends family physicians' "critical role" in caring for children and adolescents. "Collaboration is an important option for the future," he asserts. 